\title{
Modelo de control estratégico para el sector saneamiento en el Perú
}

\author{
Recepción: Julio de 2006 / Aceptación: Octubre de 2006
}

(1) Henry Humanchumo Venegas

\section{RESUMEN \\ El artículo trata sobre un modelo de control estratégico y el análisis de los indicadores estandarizados para una muestra de empresas de saneamiento en el Perú. Importante propuesta para estas organizaciones que se encuentran en un caos económico y financiero.}

Palabras Clave: Control estratégico, control clásico.

A STRATEGIC CONTROL MODEL FOR THE sanitation sector in Peru ABSTRACT

This article deals about the strategic control model and the analysis of the standardized indicators for a sample of sanitation companies in Peru. It is a very important proposal for these organizations that are in an economic and financial chaos.

Key words: Strategic control, classic control.
I N T ROD U C C I Ó N

Las empresas de saneamiento están conformadas por 46 organizaciones distribuidas en todo el territorio nacional, constituyéndose en monopolios de servicio público y en donde el gobierno es el principal accionista. Los servicios de saneamiento que se brindan a los usuarios son deficientes y la imagen que proyectan las organizaciones de saneamiento hacia la comunidad es cada vez más deteriorada. Como consecuencia de no existir un mecanismo de control estratégico para su supervisión, evaluación y monitoreo por parte de la SUNASS; haciendo fácil su politización, burocratización; encontrándose en un caos económico y financiero.

En el actual contexto que encuentran estas organizaciones se diseña un modelo de control estratégico moderno y dinámico el que deberá medir los resultados desde el punto de vista cuantitativo y cualitativo teniendo como el principal impulsor para el cambio la perspectiva de personal el que impulsará a la perspectiva de procesos para la revisión y mejora de los procesos de producción, comercial y administrativos esto permitirá cambiar la perspectiva del cliente vista desde el punto del marketing estratégico, a través de la reducción de costos en los servicios, mejor atención de los clientes y el cambio de la imagen con respecto a la empresa, y como consecuencia se tendrán mejores indicadores.

La tendencia a futuro es convertir las empresas de saneamiento en empresas modernas, eficientes y eficaces a través de la privatización, la concesión y la reestructuración, para luego venderlas o en su defecto ser compradas por las empresas de saneamiento transnacionales de agua y saneamiento que cada vez están conquistando el mercado de saneamiento como son los casos de Chile, Bolivia y Argentina. La guerra de los países en los próximos años será por el agua debido a su escasez y calidad, siendo el Perú uno de los países con este potencial hídrico.

MARCO TEÓRICO

Teorías

Basado, en las teorías explicativa de la administración [2], como la teoría neoclásica, teoría burocrática, la teoría de desarrollo organizacional y la teoría contingencial, se explicará la realidad problemática de las empresas de servicio de saneamiento en el Perú, las que vienen afrontando problemas de gestión, de imagen institucional, de exceso de personal y de restricciones financieras.

(1) Egresado del doctorado en Ciencias Administrativas, UNMSM. Consultor de las empresas de Saneamiento en el Perú. Docente de Universidad Privada Antenor Orrego - UPAO, Trujillo E-mail: hhuamanchumo@hotmail.com 
La teoría Neoclásica, explica que sin una adecuada planeación responsable y control estratégico en las empresas de servicio de saneamiento, no se logrará obtener los resultados y las metas esperadas. Se debe partir por la búsqueda de la maximización de beneficios tanto del cliente como de la entidad prestadora de saneamiento. Orientándolo principalmente a la satisfacción de los clientes quienes son los usuarios que utilizan los servicios y como consecuencia final dependerá la rentabilidad de la empresa. Esta teoría también afirma, que sin una adecuada capacitación y entrenamiento al personal en toda la estructura organizacional no se podrá exigir los rendimientos esperados a los trabajadores.

La teoría Burocrática, explica que siendo entidades únicas en el sector dentro de su jurisdicción hacen que estas se conviertan en monopolios del servicio de agua y desagüe. Otra de las razones es que al encontrarse tan reglamentadas y con engorrosos procedimientos administrativos para la obtención de nuevos servicios o las reposiciones de estos, hacen que estas instituciones no sean apreciadas como eficientes dentro de la población. Por eso es necesario hacer reingeniería, reestructuración de sus procesos y procedimientos comerciales y de producción. Así como determinar los cuellos de botella de este tipo de empresas. Esto hace que la estructura orgánica, se encuentre burocratizada con exceso de personal y como consecuencia los costos de producción y de comercialización de los servicios sean elevados.

La teoría de desarrollo organizacional, explica como estas instituciones nacen en una jurisdicción o territorio y específicamente dentro de una provincia y posteriormente se expande hacia sus localidades o zonas periféricas, surgidas como consecuencia de nuevas y urgentes necesidades del servicio de agua y desagüe. Por eso es necesario que las empresas de saneamiento deban ser analizadas en qué etapa de desarrollo se encuentran, pioneras o de nacimiento, de reglamentación, burocratización y readaptación.

La teoría contigencial, explica como estas instituciones se ven influenciadas por el entorno, la incertidumbre, la tecnología, la estrategia y la capacidad del personal esto hará que se eleve la eficacia y eficiencia de las organizaciones de saneamiento. Esto se reflejará en el diseño y rediseño de sus organizaciones de allí que se clasifiquen las organizaciones en pequeñas, medianas y grandes empresas con diferentes tipos de diseño organizacionales. El exceso de personal en todas las estructuras orgánicas y la tecnología aplicada a las áreas criticas de comercialización y producción no son las adecua- das a su realidad. Otro de los problemas son los sistemas de información y el control obsoleto, para la toma de decisiones. Ante este problema han optado por la tercerización de los servicios de saneamiento.

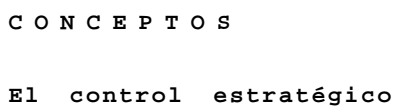

Es un sistema que establece puntos de referencia, reglas, métodos e indicadores para medir la congruencia, el avance, la eficiencia, eficacia y efectividad en el logro de las metas estratégicas y permite además una mejor comprensión de las crisis de las organizaciones. Teniendo como base el planeamiento estratégico y que está integrado por un conjunto de mecanismos tecnológicos o no de la informática cuyo objetivo es influir en los resultados del Plan. Para ello se establece puntos de referencia para medir la congruencia y avance hacia las metas, la utilización eficiente, eficaz y efectiva de los recursos, la exactitud de la información financiera, recolectando además la información real de la empresa para la subsecuente toma de decisiones que establecerán los ajustes del Plan Táctico o plan operativo (corto plazo) y del plan estratégico (largo plazo).

El control clásico

El concepto clásico, está orientado a buscar detalles de lo ocurrido en el pasado y tiene una fuerte connotación contable, por cuanto se circunscribe a la salvaguarda de los activos y a la confiabilidad de los reportes financieros. En nuestro medio es el concepto o enfoque más frecuente. El control clásico desarrolla su esfuerzo en hacer perfecto o perfeccionar el ayer, se centra en lo contable, probablemente por una mala definición de su objetivo fundamental.

EL SECTOR SANEAMIENTO EN EL PERÚ

Antecedentes

El servicio de agua potable y alcantarillado, tradicionalmente ha sido conceptuado como servicios de carácter público. El Estado asumía los gastos derivados de la prestación y los usuarios pagaban una "pensión" que en la práctica tenía el carácter de contribución.

En los años 1980, al crearse el Servicio Nacional de Abastecimiento de Agua Potable y Alcantarillado SENAPA, se constituyó una especie de holding empresarial, donde se administró y operó los sistemas de saneamiento de todas las ciudades importantes del país, se introdujo el enfoque empresarial característico de la empresa pública, utilizó una serie de subsidios entre sus unidades operativas y subsidió 
directamente a la inversión generando distorsiones en los incentivos los cuales deben ser propios a la gestión de una empresa.

A finales de los años 80, el gobierno dispuso la liquidación de SENAPA y los servicios fueron transferidos a las municipalidades, a su vez éstas constituyeron las empresas de servicios municipales dedicadas exclusivamente a la prestación de los servicios de saneamiento. Durante aproximadamente 4 años, la prestación se realizó sin un marco legal que regulara las condiciones de operación, los derechos y obligaciones de las empresas y sus clientes, la determinación de las tarifas y los estándares del servicio.

En 1994, se promulga la Ley General de Servicios de Saneamiento, Ley 26338 y su reglamento, D.S. 0995-PRES. En estos dispositivos, por primera vez en el Perú, se establece un marco legal que regula la prestación de los servicios de saneamiento.

\section{El sector saneamiento}

El marco legal define cuatro estamentos dentro del sector saneamiento, el primero fue el Órgano Rector a cargo del Ministerio de Vivienda y Construcción, cuyas principales funciones son las de formular las políticas y dictar las normas para la prestación de los servicios. El segundo, lo constituye la Superintendencia Nacional de Servicios de Saneamiento, SUNASS, como un órgano regulador de la prestación de los servicios; tal condición conlleva la aplicación de principios económicos relacionados con el uso óptimo de los recursos, se trata de bienes económicos los cuales son escasos, por lo tanto, requieren ser valorados adecuadamente tanto por los productores del servicio como por los consumidores y, cuya explotación debe darse procurando la conservación de las fuentes de agua, en armonía con el ambiente. Un tercer estamento, lo constituyen las municipalidades, en mérito a ser las beneficiarias de la transferencia de activos de SENAPA, pues son las accionistas en las empresas municipales prestadoras de servicios de saneamiento. Además, el nuevo marco legal les reconoce la facultad de otorgar el denominado derecho de explotación a los prestadores sean estos públicos, privados o mixtos. Finalmente, el cuarto, es el marco que regula los servicios de saneamiento, reconoce a los prestadores de servicios (EPS) como los entes responsables de construir, operar, mantener y administrar la infraestructura de saneamiento así como comercializar los servicios.

Situación actual de las empresas de sa neamiento

Históricamente en el Perú se ha entendido por Saneamiento Básico a la prestación de servicios de agua potable y desagüe. Luego de diez años de supuestas reformas, el Perú se compara desfavorablemente con el resto de los países sudamericanos y tan solo el $69 \%$ de la población tiene acceso a un inadecuado servicio de saneamiento y solamente mejor que Bolivia y Venezuela. Actualmente solamente 2 empresas prestadoras de servicios, proveen las 24 horas del día, pero adicionalmente reflejan pobres indicadores financieros de las EPS.

En Perú, entre los años 2000 y el 2004 habían 46 empresas municipales de saneamiento dedicadas a la prestación de servicios de saneamiento dentro del ámbito urbano de su jurisdicción. las empresas prestadoras de saneamiento se encuentran clasificadas en grandes empresas, medianas y pequeñas empresa. Fuera de esta clasificación se encuentra SEDAPAL [9].

Otro de los aspectos de análisis, es la asimetría existente entre las fuentes disponibles de agua dulce y la distribución de la población. En tanto, hacia la zona costera fluye apenas el $2 \%$ de las aguas superficiales, en esta zona se asienta el $53 \%$ de la población total del país y concentra alrededor del $85 \%$ de la actividad industrial, la actividad agrícola totalmente dependiente del riego y con un bajo nivel de tecnificación; en general con escasez de fuentes superficiales de agua, lo cual ha llevado a un alto grado de sobre explotación de las aguas subterráneas. En contraposición, hacia la vertiente oriental fluye el $98 \%$ de las aguas superficiales hacia las regiones de sierra y selva donde la población es poco concentrada.

Las leyes vigentes reconocen a los usos poblacionales, derechos de agua preferentes sobre otros usos alternativos por los cuales se paga una tarifa determinada mediante resolución suprema.

En el Perú existen aproximadamente 28.0 millones de habitantes, de los cuales el $31 \%$ viven en comunidades menores a 2 mil habitantes y el $69 \%$ restante en comunidades urbanas; de estos últimos aproximadamente 2.3 millones se encuentran habitando en ciudades cuyos servicios se encuentran administrados por municipios o empresas aun no reconocidas por SUNASS; las restantes 17.0 millones de personas se encuentran en ciudades atendidas por 46 EPS reconocidas por SUNASS, de las cuales 45 son municipales y una, SEDAPAL, entidad que está bajo la responsabilidad del gobierno central.

En términos generales para el ámbito atendido por las EPS reconocidas por la SUNASS, la cobertura de los servicios de agua potable y alcantarillado llega al $79.3 \%$ y $68 \%$ respectivamente. La continuidad en promedio es de 16.3 horas de suministro de agua potable 
por día, a pesar de que la mayor parte de empresas producen lo suficiente como para brindar un suministro continuo. Aparte de una considerable pérdida de masa de agua, el nivel de venta llega sólo hasta al $52.4 \%$ del agua producida, es decir, de cada 100 metros cúbicos que se producen, solo 52.4 llegan a facturarse. Sumándose a esto las empresas no son eficientes en la recaudación, pues arrastran una deuda equivalente a 6.2 meses de facturación (unos 150 millones de dólares aproximadamente). Estos resultados se explican por una deficiente gestión empresarial de parte de organizaciones institucionalmente muy débiles, sujetas a una fuerte injerencia política de las autoridades municipales, con una alta rotación de gerentes (en promedio un gerente general es cambiado a los 17 meses), con personal poco capacitado y mal remunerado. Crisis que ha castigado duramente la rentabilidad de las empresas al extremo que la mayor parte de ellas hace frente a deudas que las obligan más allá de sus posibilidades.

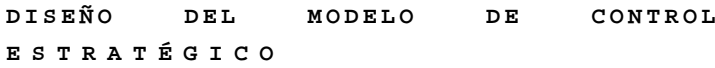

El modelo de control estratégico moderno para las empresas de saneamiento es vista desde cinco perspectivas de la organización: desde el entorno, del personal, del marketing, de los procesos y financiero. Teniendo como sustento teórico la evolución del control estratégico desde Glover, Goets, Goold y Quinn, Mintzberg, Simons, Pumpin hasta a Kaplan y Norton.

El modelo de control estratégico, es de concepción holística y dinámico se encuentra concebido por cinco perspectivas de la organización y cuatro resultados estratégicos: entre las perspectivas tenemos la del entorno, del personal, de los procesos, la del marketing y la financiera.

\section{Perspectiva del entorno ¿Qué esperamos y esperan del medio ambiente?}

La falta de consonancia entre medio ambiente y la organización hace que se vuelvan cada vez menos eficientes y eficaces. El entorno influye en el diseño y rediseño continuo de nuestra estructura orgánica. Así como de las estrategias propuesta por la organización. Entre las variables consideradas tenemos la tecnológica y el uso de tecnologías limpias, la demanda de servicios producidos con tecnologías no contaminantes y la incertidumbre del sector. Entre los que destacamos el nivel de riesgo del ingreso de competidores potenciales y visión social e integradora con la comunidad.

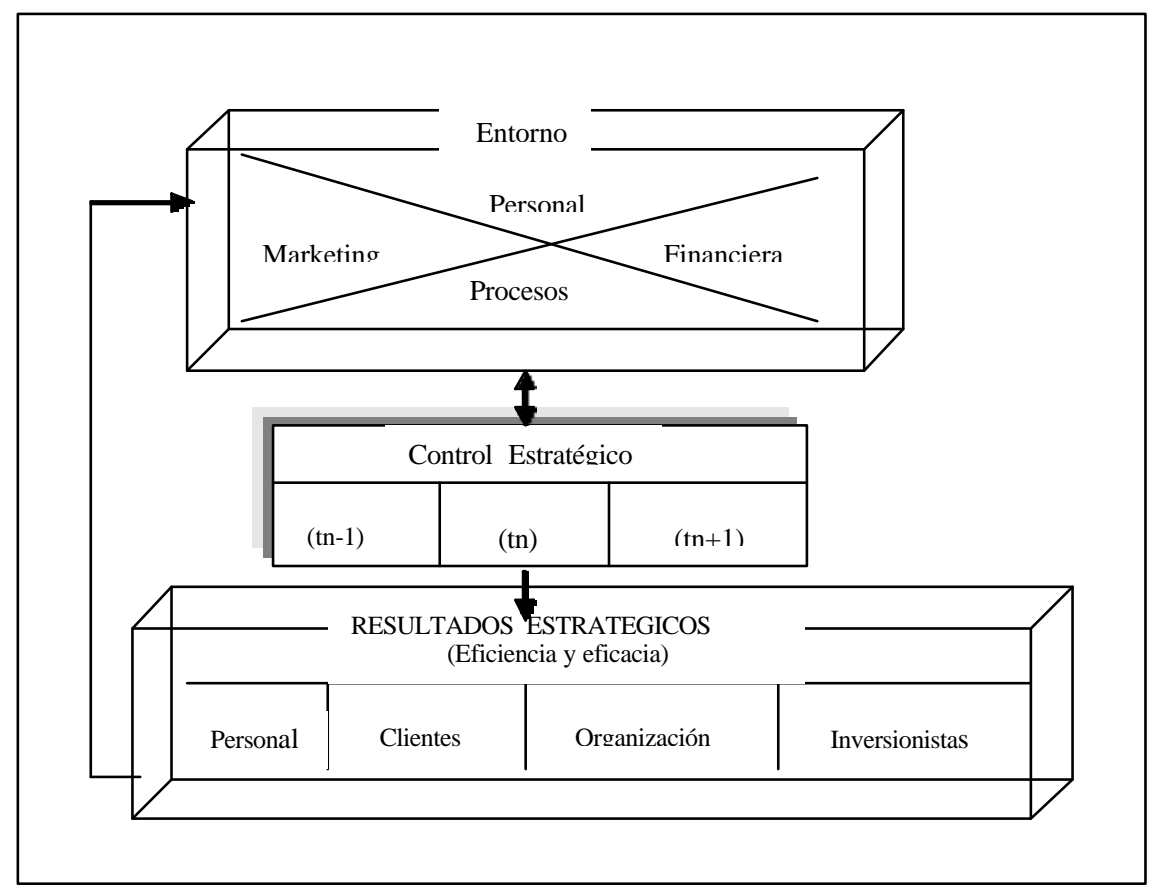

Figura 1. Perspectivas de la organización Fuente: Elaboración propia, 2006 
Cuadro 1. Indicadores del modelo de control estratégico.

\begin{tabular}{|c|c|}
\hline PERSPECTIVAS & INDICADOR \\
\hline Financiera & $\begin{array}{l}\text { EVA (valor económico agregado) } \\
\text { EBITDA (utilidad antes de impuesto y deprec.) } \\
\text { Flujo de Caja } \\
\text { Costo operacional por metro cúbico } \\
\text { Tarifa promedio por metro cúbico agua } \\
\text { Volumen de agua vendida } \\
\text { Capital de trabajo } \\
\text { Inversión en agua y desagüe }\end{array}$ \\
\hline Marketing & $\begin{array}{l}\text { Población con acceso agua /desagüe } \\
\text { Calidad del servicio } \\
\text { Empleados amables y serviciales } \\
\text { Cobranza/ Facturación anual } \\
\text { Demoras de los pagos }\end{array}$ \\
\hline Procesos & $\begin{array}{l}\text { Mejora del sistema de información } \\
\text { Gestión de inventario } \\
\text { Tasa de retornos de los activos } \\
\text { Mejora de los procesos de producción } \\
\text { Racionalización del personal }\end{array}$ \\
\hline Personal & $\begin{array}{l}\text { Habilidad de liderazgo } \\
\text { Visión integradora con la organización } \\
\text { Capacitación }\end{array}$ \\
\hline Entorno & $\begin{array}{l}\text { Ingreso de competidores potenciales } \\
\text { Gestión del medio ambiente } \\
\text { Visión integradora con la comunidad } \\
\text { Uso de tecnología limpias }\end{array}$ \\
\hline
\end{tabular}

2. Perspectiva del personal ¿Qué se debe continuar mejorando?

Después de haber analizado el entorno, rediseñado nuestra organización y ajustado nuestra estratégica el siguiente punto siguiente será el entrenamiento, capacitación y motivación de nuestro personal. La competencia es feroz en este nuevo milenio, por ello la empresa debe ser apta para innovar y mejorar. Los productos o servicios cumplen su ciclo de vida y es necesario disponer de unos nuevos, con capacidades mayores y atractivas.

3. Perspectiva de los procesos: ¿en qué podemos destacarnos?

¿Qué hacer dentro de la empresas para cumplircon las expectativas de los clientes? Los Procesos de la empresa deben estudiarse y evaluarse para conseguir la satisfacción de los consumidores.

\section{Perspectiva del Marketing: ¿qué esperan de} la empresa?

El buen Servicio al cliente es muy importante y es la base para poder permanecer en un mercado competitivo. Es probable que la Misión destaque sobre este particular. Los clientes esperan pro- ductos o servicios de óptima calidad, con un costo adecuado, que se entreguen a tiempo y que su rendimiento sea el convenido. Así la revisión desde el aprovisionamiento o el sistema logístico, la revisión de los canales de comercialización, las redes de distribución y los puntos de ventas.

5. Perspectiva Financiera: ¿Qué esperan los accionistas?

Quienes invierten su dinero esperan, en forma legítima un rendimiento adecuado. Si esto no se complace, es probable que inviertan su dinero en una empresa diferente.

El modelo de control estratégico propuesto permitirá hacer un control estratégico en tres dimensiones:

- El primer control, control estratégico denominado(CE) (tn-1) es el control pasado después haber ocurrido las operaciones referente a período(s) o proceso(s) de producción. No solamente del control de los estados financieros, si no de las otras cuatro perspectiva.

- El segundo Control, denominado por CE(tn), es el control de gestión de las operaciones diarias, en- 


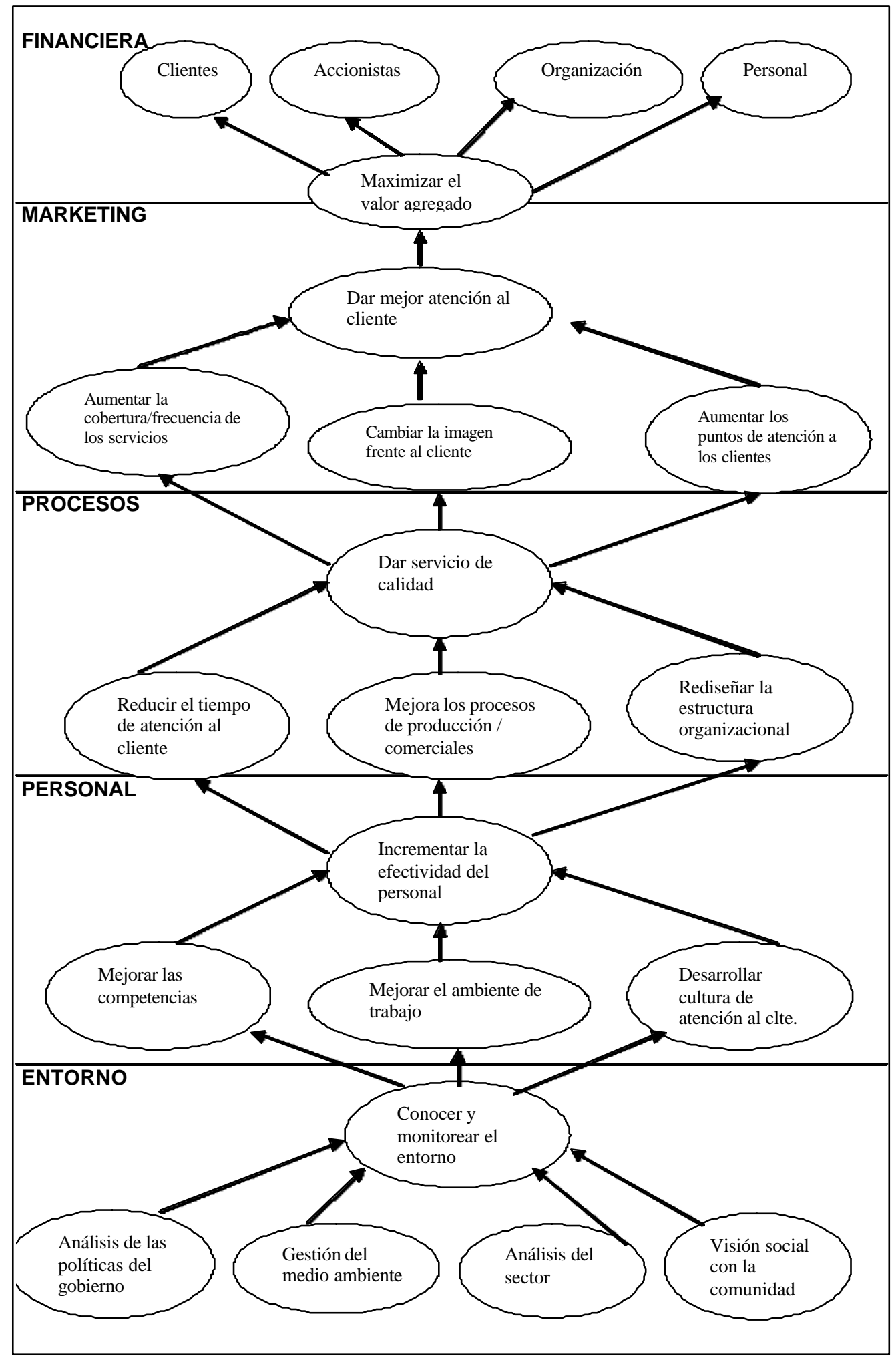

Figura 2. Indicadores del modelo de control estratégico Fuente: Elaboración propia, 2006 
focada desde punto de vista de las variables críticas. También se conoce como el control gestión de los gerentes desde el punto de vista de la toma de decisiones, de los resultados obtenidos y de su actuación vista por los accionistas o inversionistas.

- El tercer Control, $C E(t n+1)$, es el control estratégico planteado, a través de metas propuestas versus los resultados obtenidos, vista de diferentes escenarios propia de la organización, de la competencia y de las tendencias del sector.

El modelo, a través de la eficiencia y eficacia buscará los siguientes resultados estratégicos:

- Para el personal, que las personas sean consideradas como el elemento actuante, participante y de propuestas, para lo cual no solamente debe ser remunerado económicamente bien, sino motivado por diferentes formas.

- Para los clientes, que éstos sientan que se encuentran tan identificados con la organización, para lo cual se debe aplicar técnicas como de la fidelización de clientes, donde por nuestros productos o servicios que ofertamos se sientan tan satisfechos, que volverían a comprar en nuestra organización.

- Para la organización, lograr la eficiencia y eficacia. Donde habrá resuelto su problema de gastos y rentabilidad.

- Para los inversionistas, haber obtenido rentabilidad o resultados esperados de acuerdo al plan estratégico propuesto por la compañía.

AVANCE DEL PROYECTO DE INVESTIGACIÓN

El modelo de control estratégico de saneamiento se encuentra en una fase piloto aplicada a una muestra de siete empresas de saneamiento consideradas como grandes empresas. Actualmente se está en la estandarización y verificación de indicadores de control estratégico comunes.
C O N C L U S I O N E S

El modelo de control estratégico, constituirá una herramienta básica para la gestión proactiva y de toma decisiones, ya que proveerá de la información interna y externa necesaria para tomar un rápido conocimiento del estado de situación actual y la probable evolución de las empresas de saneamiento.

REFERENCIAS

BIBLIOGRÁFICAS

1. Amat, Joan. Ma․ (2001). El control de gestión: Una perspectiva de dirección. $6^{\underline{a}}$ edición. Barcelona: Ediciones Gestión 2000, S.A.

2. Chiavenato, Idalberto,(2000). Introducción a la Teoría General de Administración. Mc.Graw-Hill Latinoamérica S.A, Bogotá, octava edición, pag. 215:449

3. Jacques Lambin, Jean. (2001). Marketing estratégico. Mc.Graw-Hill interamérica de España S.A, Madrid, tercera edición.

4. Kaplan, Roberty Norton, David,(2000):"COMOUTILIZAR EL CUADRO DE MANDO INTEGRAL", Barcelona, Gestión 2000, primera edición, pag. 21:85.

5. Ley No.26538. (1994). Ley General de Servicio de Saneamiento, publicada en el Diario «El Peruano» 22/07/94, pag. 1:30

6. Mintzberg, Henry y Quinn, James Brian. (2001). E Proceso Estratégico: Conceptos, contextos y casos. México: Prentice Hall Hispanoamericana, S.A

7. Porter, Michael. (2000). Ventaja Competitiva, CECSA, México, primera edición, pag. 1:287

8. PRONAP (2005). Programa de agua potable. En: http://www.pronap.gob.pe Visitado: 10/8/06.

9. SUNASS (2005). Indicadores de Benchmarking. En: http://www.sunass.gob.pe Visitado:15/7/06. 